\title{
Estudio experimental y modelado cinético de la oxidación de $\mathrm{H}_{2} \mathrm{~S}$ y su interacción con $\mathrm{NO}$
}

\author{
Juan M. Colom-Díaz, Jorge Conchello, Ángela Millera, Rafael Bilbao, María U. \\ Alzueta \\ Grupo de Procesos Termoquímicos (GPT) \\ Instituto de Investigación en Ingeniería de Aragón (I3A) \\ Universidad de Zaragoza, Mariano Esquillor s/n, 50018, Zaragoza, Spain. \\ Tel. +34-876555451, e-mail: juanmcd@unizar.es
}

\begin{abstract}
El trabajo realizado tiene como objetivo el estudio experimental y de modelado cinético de la oxidación de sulfuro de hidrógeno $\left(\mathrm{H}_{2} \mathrm{~S}\right)$, a presión atmosférica y en un intervalo de temperaturas de 550-1100 ${ }^{\circ} \mathrm{C}$. Los resultados han sido simulados con el software Chemkin-Pro usando un mecanismo detallado de reacción compilado en este trabajo.
\end{abstract}

\section{Introducción}

Hoy en día, el incremento de la importancia de fuentes de energía no convencionales, como gas natural ácido o gas de fracking, ha despertado el interés en el uso de estos recursos sin la utilización de caros procesos de desulfuración. El sulfuro de hidrógeno puede encontrarse en pozos de gas natural (hasta un $30 \%$ de contenido de $\mathrm{H}_{2} \mathrm{~S}$ en volumen) y es un subproducto en variedad de procesos industriales [1]. Sin embargo, la naturaleza de la conversión de este compuesto sigue siendo desconocida en muchos procesos. En este contexto, el principal objetivo de este proyecto es estudiar la conversión de sulfuro de hidrógeno en condiciones de combustión, para poder predecir qué condiciones de operación son prometedoras para asegurar una combustión estable, a la vez que minimizar las emisiones contaminantes.

\section{Método experimental}

En el presente estudio lleva a cabo la oxidación de $\mathrm{H}_{2} \mathrm{~S}$ bajo distintas atmósferas reaccionantes: en condiciones oxidantes $(\lambda=1.2)$, estequiométricas $(\lambda=1)$ y condiciones reductoras $(\lambda=0.7, \lambda=0.3)$. Se considera la reacción de oxidación: $\mathrm{H}_{2} \mathrm{~S}+\frac{3}{2} \mathrm{O}_{2} \rightarrow$ $\mathrm{SO}_{2}+\mathrm{H}_{2} \mathrm{O}$, para calcular la estequiometría, $\lambda$. Los experimentos se realizan a presión atmosférica, en el intervalo de temperaturas $550-1100{ }^{\circ} \mathrm{C}$ y en un reactor de flujo de cuarzo con una zona de reacción de $8.7 \mathrm{~mm}$ de diámetro interno y $200 \mathrm{~mm}$ de longitud. El caudal total en todos los experimentos es de $1 \mathrm{~L}(\mathrm{STP}) / \mathrm{min}$, y el tiempo de residencia de los gases en función de la temperatura de 195/T(K) en segundos. Los gases reactantes son $\mathrm{H}_{2} \mathrm{~S}, \mathrm{O}_{2}$, NO y nitrógeno para completar el caudal total. Además de $\sim 1 \%$ de vapor de agua, lo cual se consigue saturando la corriente de nitrógeno a través de un borboteador. De esta forma, se evitan reacciones de terminación en las paredes del reactor. A la salida de éste se interrumpe la reacción con una corriente de refrigeración que disminuye drásticamente la temperatura, para congelar la reacción. La concentración de $\mathrm{H}_{2} \mathrm{~S}$ es de 500 ppm en todos los experimentos. Para cuantificar $\mathrm{H}_{2} \mathrm{~S}$ e $\mathrm{H}_{2}$ se utiliza un cromatógrafo de gases, un analizador en continuo con sensor UV para $\mathrm{SO}_{2}$ y un analizador en continuo con sensor IR para NO.

\section{Modelo cinético}

Los resultados experimentales se analizan mediante un modelo cinético con el software Chemkin-Pro y utilizando el modelo de reactor flujo pistón. Para llevar a cabo la simulación se precisa de una serie de reacciones entre $\mathrm{H}_{2} \mathrm{~S}, \mathrm{O}_{2}$ y $\mathrm{NO}$, que se agrupan en el llamado mecanismo de reacción, y que cuentan con los correspondientes parámetros cinéticos. El mecanismo base usado puede encontrarse en detalle en [2], el cual está actualizado en este trabajo con un sub-mecanismo para la conversión de $\mathrm{H}_{2} \mathrm{~S}$ de la bibliografía [3], y que incluye especies como $\mathrm{SH}$, $\mathrm{OH}$ y $\mathrm{HO}_{2}$ de importancia en el sistema de oxidación de sulfuro de hidrógeno.

\section{Resultados}

Los resultados obtenidos indican que la conversión de $\mathrm{H}_{2} \mathrm{~S}$ aumenta con una mayor presencia de oxígeno, con una temperatura de inicio de reacción en torno a los $650{ }^{\circ} \mathrm{C}$ en todos los casos, independientemente de la estequiometría, Figura 1. La presencia de $\mathrm{NO}$ desplaza la oxidación de $\mathrm{H}_{2} \mathrm{~S}$ a temperaturas más bajas en condiciones oxidantes, mientras que en condiciones estequiométricas y 
reductoras el proceso se desplaza a temperaturas más altas. La concentración de NO de entrada se ve prácticamente inalterada durante todos los experimentos. El balance de azufre no se cumple en condiciones en las que hay un déficit de oxígeno. Esto se atribuye a la formación de azufre, lo cual fue constatado, pero no cuantificado, al observar un depósito sólido amarillo a la salida del reactor. En la Figura 2 se puede observar un ejemplo de la simulación con el modelo cinético para uno de los experimentos. En general, las simulaciones predicen bien las tendencias experimentales.

\section{Conclusiones y trabajo futuro}

Los experimentos muestran una mayor conversión del sulfuro de hidrógeno con atmósferas ricas en oxígeno, con una temperatura de inicio de la reacción independiente de la estequiometría. La oxidación de $\mathrm{H}_{2} \mathrm{~S}$ se modifica al introducir NO. El modelo cinético simula bien los datos experimentales, por lo que podría utilizarse en el diseño y optimización de procesos en casos reales, tales como procesos de combustión y el proceso Claus.

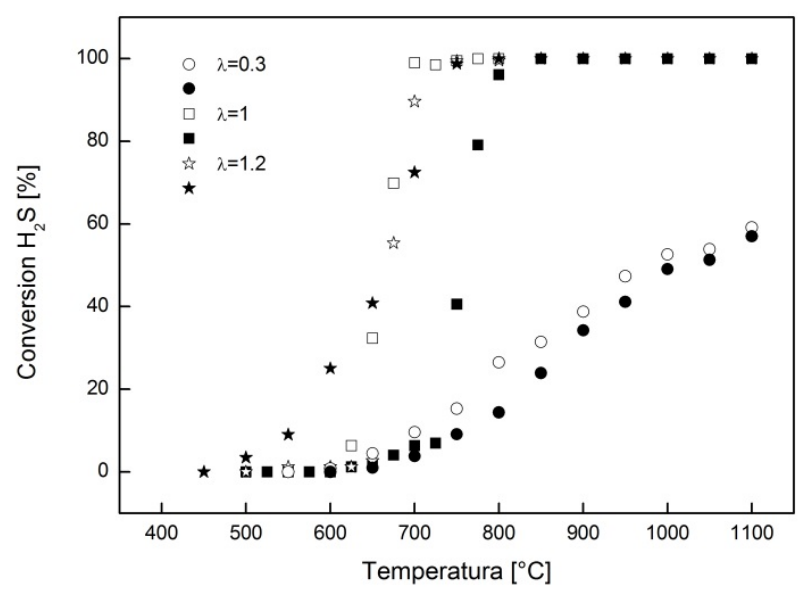

Figura 1: Conversión de $\mathrm{H}_{2} \mathrm{~S}$ frente a la temperatura para distintas atmósferas reaccionantes (puntos vacíos) y la influencia del NO para los mismos experimentos (puntos rellenos).

\section{Agradecimientos}

Los autores quieren expresar su gratitud al Gobierno de Aragón y al Fondo Social Europeo (grupo GPT), y a MINECO y FEDER (Proyecto CTQ201565226) por la financiación concedida. Juan Manuel Colom agradece a MINECO por la beca predoctoral (Beca BES-2016-076610).

\section{REFERENCIAS}

[1]. HAMMER, G., LÜBCKE, T., KETTNER, R., PILLARELLA, M.R., RECKNAGEL, H., COMMICHAU A., NEUMANN, H.J.,

PACZYNSKA-LAHME, B. Ullmann's Encyclopedia of Industrial Chemistry. 2012, vol. 23, Wiley-VCH, Weinheim, p. 740.

[2]. ABIÁN, M., MILLERA, Á., BILBAO, R., ALZUETA, M.U. Impact of $\mathrm{SO}_{2}$ on the formation of soot from ethylene pyrolisis. Fuel. 2005, 159, 550558.

[3]. SONG, Y., HASHEMI, H., CHRISTENSEN, J.M., ZOU, C., HAYNES, B.S., MARSHALL, P., GLARBORG, P. Exploratory Flow Reactor Study of $\mathrm{H}_{2} \mathrm{~S}$ Oxidation at 30-100 Bar. International Journal of Chemical Kinetic. 2017, 49, 37-52.

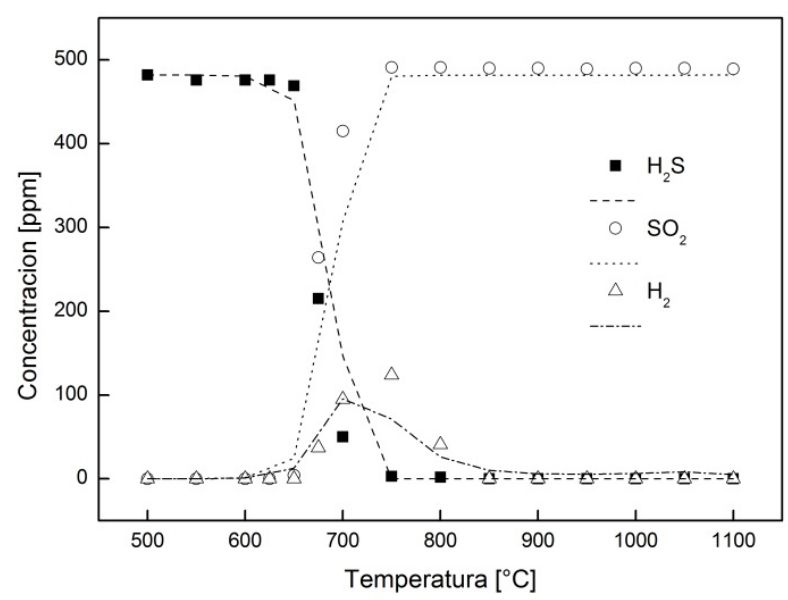

Figura 2: Concentraciones de $\mathrm{H}_{2} \mathrm{~S}, \mathrm{SO}_{2}$ y $\mathrm{H}_{2}$ para $\lambda=1.27$ en función de la temperatura. Datos experimentales (puntos) y resultados del modelo cinético (líneas discontinuas). 